\title{
Self-pumped optical delay line based on Brillouin fast light in optical fibers
}

\author{
Luc Thévenaz and Sanghoon Chin \\ Ecole Polytechnique Fédérale de Lausanne, STI-NAM Station 11, CH-1015 Lausanne, Switzerland \\ luc.thevenaz@epfl.ch, sanghoon.chin@epfl.ch
}

\begin{abstract}
An extremely simple technique is demonstrated to realize tunable delays in optical fibers controlled by the signal average power. The system self-adapts in real time to the Brillouin fiber properties and to the signal bandwidth.
\end{abstract}

(C)2008 Optical Society of America

OCIS codes: (060.4370) Nonlinear optics, fibers; (290.5900) Scattering, stimulated Brillouin; (350.5500) Propagation

\section{Introduction}

Slow \& fast light based on stimulated Brillouin scattering (SBS) in optical fibers has proved these past few years to be a very efficient and flexible approach to realize optically controlled delay lines [1-3]. Actually the exploitation of the SBS interaction is not restricted to its natural resonance characteristics as observed using a CW pumping, but its spectral characteristics can be engineered to a wide extent using a modulated pump or combining Brillouin gain and loss spectra using multiple pumps. For instance the bandwidth of the Brillouin spectral resonance can be shaped by a spectrally broadened pump to extend the bandwidth to many $\mathrm{GHz}[4,5]$ or to optimize the delaying efficiency [6,7]. The superposition of Brillouin gain and loss spectra generated by distinct pumps can produce a composite spectral resonance that can lead to optical delays with no amplitude change [8] and to a theoretically unlimited bandwidth [9].

We recently demonstrated that optical delays can also be produced using no pump at all, by simply taking advantage of the loss induced by the depletion resulting from the amplified spontaneous Brillouin emission generated by the signal itself [10]. The scheme was experimentally proved for an isolated pulse propagating in the fiber and advancements up to a 0.24 fractional delay could be obtained by just varying the signal power. The technique was further studied and developed and we here present the state-of-the-art of this self-pumped technique, in particular its adequacy to delay a real data stream and to adapt to the signal bandwidth.

\section{Principle}

It is well-known that stimulated Brillouin scattering can be a serious penalty in transmission systems, since it is observed that a substantial fraction of the power propagated in a signal lightwave can actually be transferred to a spontaneous amplified Stokes wave travelling in the opposite direction through the fiber. This causes an extra loss and actually all increments of light turns out to be totally transferred to the Stokes wave once a critical signal power is reached. This critical power is in the $1-10 \mathrm{~mW}$ range for long fibers and the signal intensity actually saturates to that value since all extra light is straightforwardly transferred to the Stokes wave.

This feature can be positively exploited to generate tunable delays, since this loss caused by the depletion from the Stokes wave is narrowband by essence and its magnitude is simply changed by incrementing the signal power. The scheme is very interesting, since in addition to the absence of a distinct pump source it contains many self-adapting features:

- The output signal power remains constant when the input signal power is varied, since it saturates at the Brillouin critical power. This way delays can be naturally generated with no change in the signal amplitude.

- The loss spectral feature due to the Stokes depletion is automatically placed at the center of the signal spectrum and naturally compensates for any drift in the signal frequency and in the Brillouin shift of the delaying fiber.

- The polarization of the Stokes waves actually matches the signal polarization for such high gains, so that the system places itself automatically in a situation of maximum efficiency as far as polarization is concerned.

- The loss spectral width also tracks the signal spectral distribution and adapts to a wide extent to the bandwidth of the signal.

This practically means that such an optical delay line can be realized by just plugging an optical signal with sufficient average power into a segment of fiber without any further adjustments. The only point that must be 


\section{STuC5.pdf}

addressed carefully is the fact that the power of the signal must be as much as possible constant when averaged over the delaying fiber to avoid any jitter in delay and amplitude. This is not a serious issue for repetitive signals, but a long fiber segment may be required to properly average a signal showing large variations in its bit sequence.
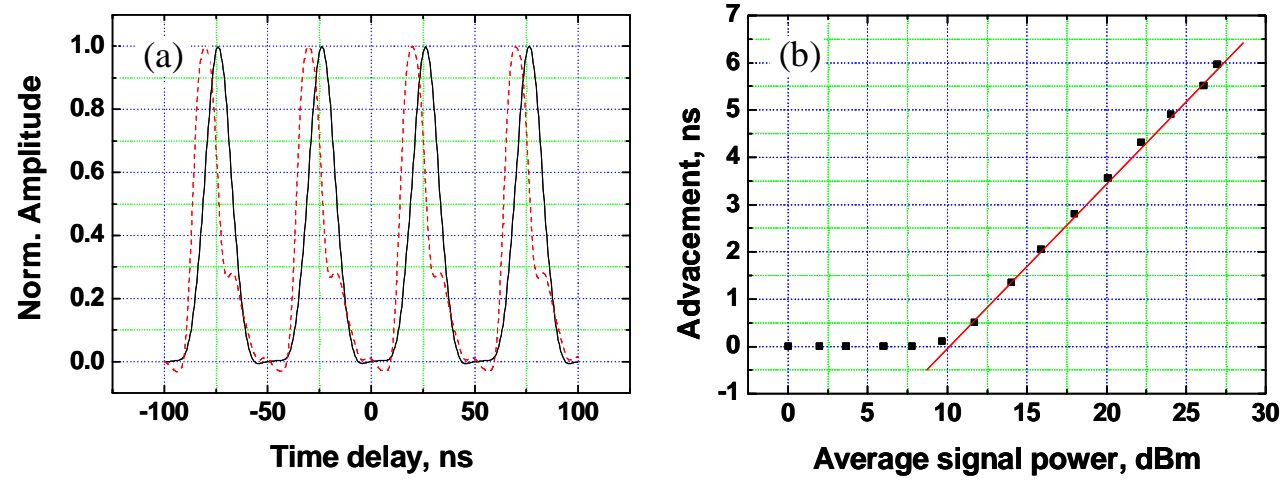

Figure 1:

(a) Temporal traces of the pulse train for a signal power below the critical power (solid line) and at maximum signal power (dashed line).

(b) Signal advancement as a function of the pulse average input power

\section{Results}

Figure 1-(a) shows the time traces obtained for a $50 \mathrm{~ns}$ pulse train showing a $30 \%$ normalized repetition rate. The DC component superimposed on the signal is about $3 \%$ of the pulse peak amplitude and has therefore a negligible impact. The maximum obtained delay is $14.2 \mathrm{~ns}$ that corresponds to a fractional delay of 0.42 . Actually the maximal delay is obtained when the amplified spontaneous Stokes emission is intense enough to generate its own Stokes wave co-propagating with the input signal.

Figure 1-(b) shows the measured advancements on the signal obtained from the peak pulse position, as a function of the average signal input power. As anticipated no timing effect is observed as long as the signal is below the Brillouin critical power of about $10 \mathrm{dBm}$ in the $12 \mathrm{~km}$ long delaying fiber. It must be pointed out that unlike classical pumped configurations the advancement is no longer proportional to the pumping power in this scheme, but depends logarithmically on the input signal power. This can be explained straightforwardly from the fact that the output power saturates above the Brillouin critical power and this corresponds to an effective induced loss for the signal that logarithmically scales with its power.

The bandwidth property of this self-pumping scheme was then tested by measuring the linewidth of the Stokes emission for increasing data rate of the signal. The signal was sequenced so that its average power is maintained constant for all data rate and the only varying parameter is its bandwidth. Figure 2 shows that the Stokes emission and thus the Brillouin loss resonance experienced by the signal - adapts its spectral width proportionally to the signal bandwidth. Nevertheless this spectral width remains a fraction of the signal bandwidth, corresponding to approximately $45 \%$ of the spectral coverage of a broadband signal.

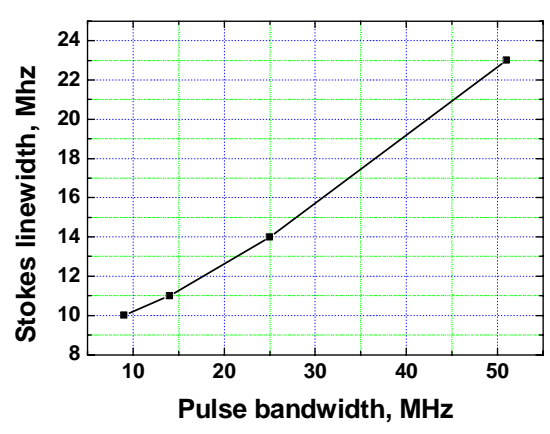

Fig.2: Measured Stokes linewidth as a function of the measured signal bandwidth.

According to its modest requirement in optical devices and its unique self-adapting properties this configuration is certainly attractive to realize optically-controlled delay lines that are easy to implement, cost effective and radically simple.

\section{References}

[1] K. Y. Song, M. Gonzalez Herráez and L. Thévenaz, Opt. Express 13, 82-88 (2005).

[2] Y. Okawachi et al, Phys. Rev. Lett. 94, 153902 (2005).

[3] M. G. Herráez, K. Y. Song and L. Thévenaz, Appl. Phys. Lett., 87, 081113 (2005).

[4] M. G. Herráez, K. Y. Song and L. Thévenaz, Opt. Express, 14, 1395-1400 (2006).

[5] Z. Zhu et al, J. Lightwave Technol. 25, 201-206 (2007).

[6] Zadok, O. Raz, A. Eyal and M. Tur, Photon. Tech. Lett., 19, 462-464 (2007).

[7] R. Pant, M. D. Stenner, M. A. Neifeld, and D. J. Gauthier, Opt. Express 16, 2764-2777 (2008)

[8] S. Chin, M. Gonzalez Herráez and L. Thévenaz, Opt. Express 14, 10684-106924 (2006).

[9] K. Y. Song and K. Hotate, Opt. Lett. 32, 217-219 (2007).

[10] S. Chin, M. Gonzalez-Herraez, and L. Thévenaz, in Slow and Fast Light, OSA Technical Digest (CD) (Optical Society of America, 2007), paper SWC5. 